hospital were recruited. The patients were divided into three groups according to their risk factors. The patients without hypertension and diabetes were included into Group $A(n=95)$. The patients with high blood pressure or diabetes were included into group $B(n=221)$ and the patients with both of high blood pressure and diabetes were included into group $C(n=72)$. Coronary artery calcification score, lipid profiles (lipoprotein (a), LDL, HDL, TG, TC) and coronary angiography were determined in each group.

Results Among the 3 groups, there is no significant difference between sex, drinking history, smoking history; there is significant difference between age and the incidence of coronary heart disease among the 3 groups (age $\mathrm{F}=5.737, \mathrm{p}=0.005$; coronary heart disease $\mathrm{F}=6.283, \mathrm{p}=0.002$. Coronary artery calcification score is significantly higher in group C than that of groups A (groups C 256.9 \pm 430.199 VS group A 103.74 $\pm 299.85, p=0.011)$. Coronary artery calcification score was positively correlated with lipoprotein (a) $(p=0.005)$, age $(p=0.021)$ in group A. Coronary artery calcification score was positively correlated with low-density lipoprotein $(p=0.018)$, age $(p=0.000)$ in group $B$. There is no significantly correlation between coronary artery calcification score and lipid profiles in group C. Summary analysis coronary artery calcification score was positively correlated with LP (a) $(p=0.013)$, low-density lipoprotein $(p=0.021)$, age $(p=0.000)$.

Conclusion In the low-risk coronary heart disease group, lipoprotein (a) is positively correlated with coronary calcification score, which suggests lipoprotein (a) is an independent risk factor for coronary artery calcification for these apparently low risk patients. The present study may contribute to the early diagnosis and intervention of coronary artery disease for those patients.

\section{C0346 SCREENING OF SLEEP APNOEA-HYPOPNOEA SYNDROME FROM ECG DERIVED RESPIRATION OF AMBULATORY ECG}

doi:10.1136/hrt.2010.208967.346

\begin{abstract}
${ }^{1}$ Guangming Tong Guangming Tong, ${ }^{2}$ Haicheng Zhang Haicheng Zhang, ${ }^{2}$ Jihong Guo Jihong Guo, ${ }^{3}$ Fang Han Fang Han. ${ }^{1}$ Department of Cardiology The Second Hospital Affiliated To Soochow University; ${ }^{2}$ Department of Cardiology, Peking University Peoples Hospital; ${ }^{3}$ Sleep Center of Peking University Peoples Hospital
\end{abstract}

Objective To evaluate the feasibility of screening sleep apnoeahypopnoea syndrome (SAHS) from ECG-derived respiration (EDR) of ambulatory ECG (AECG) monitoring.

Methods The overnight sleep investigation was administered to 80 subjects by polysomnogram (PSG) and $24 \mathrm{~h}$ AECG monitoring simultaneously during February through November, 2004. The ECG analysers did not know the PSG results at all. They were both asked to give the apnoea hypopnoea index (AHI) by EDR and PSG respectively. The PSG result was considered as the gold standard so as to evaluate the feasibility of screening SAHS from EDR of AECG monitoring.

Results The average age, male gender, body mass index, history of hypertension were higher in the SAHS $(+)$ patients than those of the SAHS (-) patients. Automatic analysis was performed with software in a sensibility of $75 \%, 87.5 \%$ and $100 \%$ respectively. When software sensibility adjusted to $75 \%$, the sensitivity of screening SAHS with EDR was $26.7 \%$, with the specificity of $80 \%$, the positive predictive value of $80 \%$, the negative predictive value of $26.7 \%$, the diagnose accordance rate of $40 \%$. When software sensibility was adjusted to $87.5 \%$, the sensitivity of screening SAHS with EDR was $55 \%$, with the specificity of $45 \%$, the positive predictive value of $75 \%$, the negative predictive value of $25 \%$, and the diagnose accordance rate of $52.5 \%$. When software sensibility was adjusted to $100 \%$, the sensitivity of screening SAHS with EDR was $88.3 \%$, with the specificity of $35 \%$, the positive predictive value of $84.1 \%$, the negative predictive value of $50 \%$, and the diagnose accordance rate of $75 \%$.
Conclusion EDR technique of AECG was useful to screen the suspicious SAHS patients, sensitivity and the diagnosis coincidence rate was higher when the sensibility of automatic analysis software was adjusted to $100 \%$.

\section{e0347 THE CHANGES OF HEART RATE TURBULENCE (HRT) IN SLEEP APNOEA-HYPOPNOEA SYNDROME (SAHS)}

doi:10.1136/hrt.2010.208967.347

Tong Guangming, Tong Guangming. Department of Cardiology The Second Hospital Affiliated To Soochow University

Objective We investigated the changes of heart rate turbulence (HRT) in sleep apnoea- hypopnoea syndrome (SAHS).

Methods 75 patients underwent overnight polysomnography for clinically suspected SAHS and simultaneous Holter monitoring (23:00-6:00), According to the apnoea-hypopnoea index (AHI), the patients were assigned to group SAHS $(+)(\mathrm{AHI} \geq 5, \mathrm{n}=52)$ or group SAHS (-) (AHI <5, n=23). HRT (onset, slope) of two groups were compared.

Results Turbulence slope (TS) of group SAHS (+) was significantly lower in group SAHS $(-)(p<0.01)$, turbulence onset of two groups all smaller than zero, turbulence onset of group SAHS $(+)$ were higher than group SAHS (-)'s, but no significant difference, the number of ventricular premature contractions of group SAHS $(+)$ were more than group SAHS (-)'s, but also no significant difference. Conclusions Heart rate turbulence phenomenon diminishes in sleep apnoea- hypopnoea syndrome patients, indicating demages in cardiac autonomic activity in SAHS, turbulence slope decreasing could be considered as prognosis index of SAHS.

\section{e0348 THE EFFECTS OF VALSARTAN ON ANGIOTENSIN II TYPE 1 AND TYPE 2 RECEPTOR IN ISOLATED REPERFUSED ISCHAEMIC RAT HEARTS}

doi:10.1136/hrt.2010.208967.348

Zhang Yingjie, Bai Xiaojuan, Qi Zhimin, Wang Hongxin. Institute of Cardiology, the First Hospital Affiliated to Jinzhou Medical University, Jinzhou

Object To determine the effects of Angiotensin II Type 1 receptor blockade valsartan on $\mathrm{AT}_{1}$ and $\mathrm{AT}_{2}$ receptor during ischaemia reperfusion

Methods The hearts of 24 SD rats were isolated, linked to Langedorff perfusion apparatus, and randomly divided into 3 equal groups: control group, perfused with modified Kreb-Henseleit (K-H) buffer for $110 \mathrm{~min}$; ischaemia/reperfusion (I/R) group, perfused with K-H buffer for $20 \mathrm{~min}$, exposed to ischaemia for $30 \mathrm{~min}$, and then reperfused with $\mathrm{K}-\mathrm{H}$ buffer for $60 \mathrm{~min}$; valsartan group, perfused with K-H buffer with valsartan for $20 \mathrm{~min}$, exposed to ischaemia for $30 \mathrm{~min}$, and then reperfused with $\mathrm{K}-\mathrm{H}$ with valsartan for $60 \mathrm{~min}$. The left ventricular (LV) function including maximal uprising velocity of left ventricular pressure $\left(+\mathrm{dp} / \mathrm{dt}_{\max }\right)$ and maximal decreasing velocity of left ventricular pressure $\left(-\mathrm{dP} / \mathrm{dt}_{\max }\right)$ were monitor. The coronary effluent were measured $20 \mathrm{~min}$ after the stabilisation of perfusion, and 20, 40, and $60 \mathrm{~min}$ after reperfusion. After the stop of reperfusion, the structure were observed using electron microscope. The $\mathrm{AT}_{1}$ and $\mathrm{AT}_{2}$ receptor mRNA express were examined by Northern blot. The $\mathrm{AT}_{1}$ and $\mathrm{AT}_{2}$ receptor protein expression were examed by Northern blot.

Results Compared with control, I/R impaired left ventricular systolic and diastolic function (+dp/dtmax $1892 \pm 231 \mathrm{~mm} \mathrm{Hg} \cdot \mathrm{s}^{-1}$ vs $836 \pm 223 \mathrm{~mm} \mathrm{Hg} \cdot \mathrm{s}^{-1}$; $-\mathrm{dp} / \mathrm{dtmax}-1175 \pm 223 \mathrm{~mm} \mathrm{Hg.s^{-1 }}$ vs $-613 \pm 224 \mathrm{~mm} \mathrm{Hg} . \mathrm{s}^{-1}$, all $\mathrm{p}<0.01$ ), decreased coronary effluent $\left(5.9 \pm 0.8 \mathrm{ml} . \mathrm{min}^{-1}\right.$ vs $\left.3.3 \pm 0.5 \mathrm{ml} \cdot \mathrm{min}^{-1}, \mathrm{p}<0.01\right)$ damaged the 